\title{
Cerebral mass in HIV infection
}

Due to an editing error, the term "progressive multifocal leucoencephalopathy" was used incorrectly twice in this Endgames quiz (BMJ 2013;347:f6314, doi:10.1136/bmj.f6314). In both the fourth paragraph of the long answer to question 3 , and the third sentence of the short answer to question 4 , "progressive multifocal leucoencephalopathy" should have read "primary central nervous system lymphoma."

Cite this as: BMJ 2013;347:f6702

๑ BMJ Publishing Group Ltd 2013 